\title{
Pseudo-anévrisme compliquant une pancréatite chronique, une cause rare d'ictère obstructif
}

\author{
Pseudoaneurysm complicating Chronic Pancreatitis, a Rare Cause of Obstructive Jaundice
}

\section{O. Peyrony $\cdot$ A. Elezi $\cdot$ P. Bourrier}

Reçu le 11 avril 2016; accepté le 4 mai 2016

(C) SFMU et Lavoisier SAS 2016

Un patient, âgé de 42 ans, aux antécédents d'éthylisme chronique compliqué de nombreux épisodes de pancréatites aiguës, consulte aux urgences pour une altération de l'état général, un ictère et une douleur épigastrique intermittente évoluant depuis trois semaines. L'examen clinique objective un ictère cutanéomuqueux franc, sans fièvre, et un abdomen souple. L'échostéthoscopie (échographie ultraportable réalisée au lit du patient) montre une dilatation de la voie biliaire principale (Fig. 1A) et des voies biliaires intrahépatiques, ainsi qu'une image kystique (arrondie et anéchogène) de la tête du pancréas qui est le siège de multiples calcifications (Fig. 1B). Le scanner confirme la dilatation des voies biliaires causée par deux pseudo-anévrismes développés aux dépens de l'artère splénique et d'une branche de l'artère mésentérique supérieure (Fig. 1C). Le patient est transféré dans un service de chirurgie vasculaire où les anévrismes ont été mis à plat. Les pseudo-anévrismes sont une complication rare mais potentiellement grave des pancréatites chroniques. Ils se forment suite à l'érosion de la paroi d'une artère, soit directement par les enzymes pancréatiques, soit par l'érosion d'un vaisseau par un pseudokyste, qui se transforme ainsi en pseudoanévrisme [1]. Les artères les plus fréquemment atteintes sont les artères splénique et gastroduodénale. La principale complication de ces pseudo-anévrismes est la rupture hémorragique intra- ou rétropéritonéale, intradigestive ou dans les canaux pancréatiques. Le traitement de première intention est alors l'artério-embolisation [2].

\footnotetext{
O. Peyrony $(\bowtie) \cdot$ A. Elezi

Service des urgences, hôpital Saint-Louis,

Assistance Publique Hôpitaux de Paris,

1, avenue Claude Vellefaux, F-75010 Paris, France

e-mail : olivier.peyrony@aphp.fr

\section{P. Bourrier}

Service de radiologie, hôpital Saint-Louis,

Assistance Publique Hôpitaux de Paris,

1, avenue Claude Vellefaux, F-75010 Paris, France
}

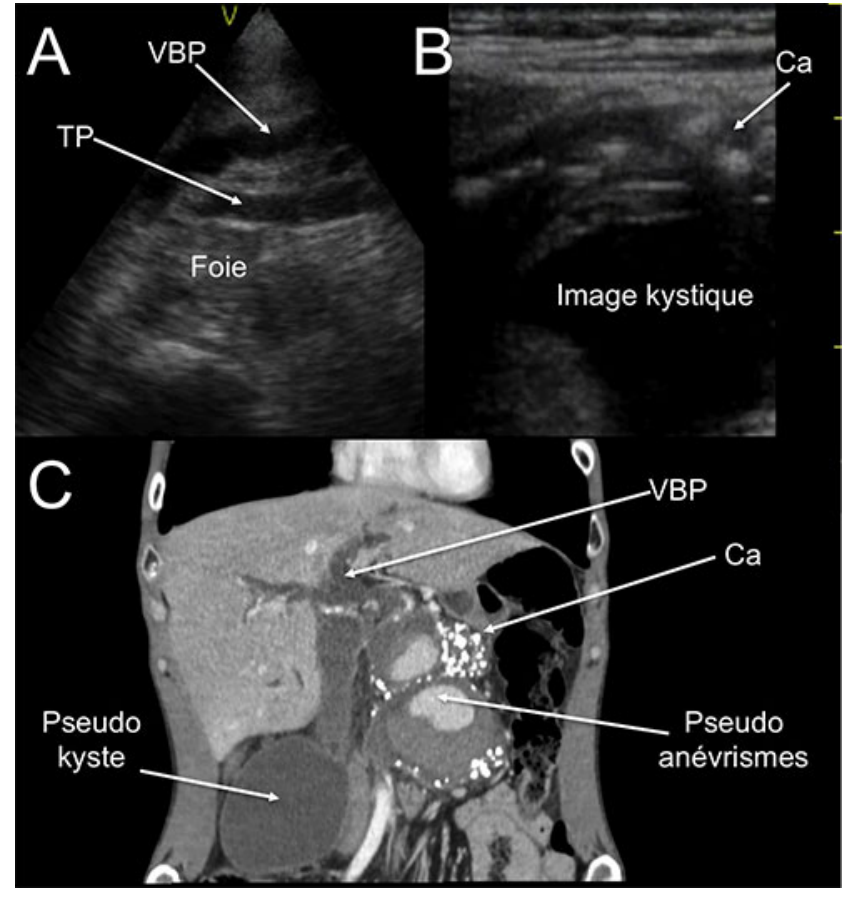

Fig. 1 Échostéthoscopie montrant une dilatation de la voie biliaire principale (VBP) qui apparaît festonnée en avant du tronc porte (TP) créant une image en « fusil hilaire » (A) et une image kystique (arrondie et anéchogène) entourée de calcifications $(\mathrm{Ca})$ sous le hile hépatique (B). Scanner (C) montrant deux pseudoanévrismes partiellement thrombosés, aux dépens de l'artère hépatique droite et d'une branche de l'artère mésentérique supérieure ainsi qu'un pseudo-kyste rétropéritonéal

\section{Références}

1. Mendelson RM, Anderson J, Marshall M, Ramsay D (2005) Vascular complications of pancreatitis. ANZ J Surg 75:1073-9

2. Kim JH, Kim JS, Kim CD, et al (2007) Clinical features of pseudoaneurysms complicating pancreatitis: single center experience and review of Korean literature]. Korean J Gastroenterol $50: 108-15$ 